\title{
Predictors of Class I epilepsy surgery outcome in tumour-related chronic temporal lobe epilepsy in adults
}

\author{
Arkadiusz Nowak ${ }^{1}$, Andrzej Rysz ${ }^{1}$, Tomasz Dziedzic ${ }^{1}$, Tomasz Czernicki ${ }^{1}$, Przemysław Kunert ${ }^{1}$, \\ Edyta Maj ${ }^{2}$, Andrzej Marchel ${ }^{1}$
}

${ }^{1}$ Department of Neurosurgery, Medical University of Warsaw, Warsaw, Poland

${ }^{2}$ 2nd Department of Clinical Radiology, Medical University of Warsaw, Warsaw, Poland

\section{ABSTRACT}

Objective. Temporal lobe tumours, especially low-grade gliomas and glioneuronal tumours, are common causes of seizures in patients referred for epilepsy surgery. We here present our experience of surgical treatment of patients with intractable chronic epilepsy associated with temporal lobe tumours, focusing on the long-term surgical outcomes and the features associated with better seizure control.

Methods. In this study, we retrospectively analysed 44 consecutive patients from a total of 182 with refractory temporal lobe epilepsy presenting with long-term intractable epilepsy due to a temporal lobe tumour who were surgically treated at our institution between 2005 and 2015 with post-surgical follow-up of at least two years. All patients underwent a standard presurgical evaluation that included: history and physical examination with a description of the seizure semiology, serial scalp EEG recording, brain MR imaging, and a detailed neuropsychological evaluation. Our surgical strategy comprised tumour resection, and combined mesial temporal and neocortical resection in most cases.

Results. No patient died during surgery or the postoperative course. Seven patients had postoperative complications, of whom two had permanent hemiparesis due to ischaemic stroke. At the final follow-up, a favourable seizure outcome (Engel Class I) was found in 37 patients (84\%), including 31 (70.5\%) in Engel Class IA (excellent result). Two (4.5\%) patients presented with an Engel Class II outcome (unfavourable outcome). Five patients (11.5\%) were in Engel Classes III or IV (surgical failure). We found that complete resection of the hippocampus along with tumour and temporal pole removal was strongly associated with seizure freedom $(p=0.015)$. Pathological diagnosis was also a significant prognostic indicator of tumour-related seizure freedom. Patients with a diagnosis of a glioneuronal tumour benefited from more seizure freedom after resection compared to those who had a low-grade glioma $(p=0.024)$.

Conclusion. The most appropriate management of tumour-related chronic temporal lobe epilepsy in adults appears to be tailored temporal lobe resection including tumour and hippocampal complex removal. Surgical treatment of tumoural temporal lobe epilepsy demonstrates excellent results in terms of seizure improvement, especially in patients with glioneuronal tumours.

Key words: seizure, intractable epilepsy, temporal lobe, tumor, surgical treatment

(Neurol Neurochir Pol 2019; 53 (6): 466-475)

\section{Introduction}

Seizures are a common manifestation of brain tumours. Tumour-related epilepsy significantly affects quality of life, and can result in significant morbidity [1-3]. Supratentorial tumours constitute $10-50 \%$ of the pathological diagnosis in patients undergoing surgery for chronic epilepsy [3, 4]. Among these, temporal lobe tumours, especially low-grade gliomas and glioneuronal tumours, are the most common causes of seizures in patients referred for epilepsy surgery [4]. In particular, glioneuronal tumours, low-grade brain tumours of glioneuronal origin that are often associated with focal cortical dysplasia (FCD), are commonly present with intractable epilepsy. Epilepsy associated with low-grade temporal lobe brain

Address for correspondence: Arkadiusz Nowak, Department of Neurosurgery, Medical University of Warsaw, Banacha 1a Str., 02-097 Warsaw, Poland.

e-mail: arkady.n@wp.pl 
tumours is generally poorly controlled by antiepileptic drugs, although it is extremely responsive to surgical treatment. Low-grade tumours associated with refractory epilepsy are generally indolent entities in which there are both oncological and epileptic aspects of neurosurgical treatment. Given the expected long-term survival of patients with low-grade tumours, achieving seizure control remains one of the most important treatment goals.

Factors associated with seizure control remain incompletely understood, and the best management strategy of tumour-related focal epilepsies has not been fully established. Some investigators have considered resection of the tumour alone to be sufficient for good seizure control [4-6], while others have advocated additional resection of epileptogenic zones adjacent to the tumour (usually the amygdala and hippocampus) to optimise seizure outcome [7-12].

We present our experience of surgical treatment of patients with intractable chronic epilepsy associated with temporal lobe tumours, focusing on the long-term seizure outcomes and the features associated with better seizure control.

\section{Material and methods}

\section{Patient population}

A total of 182 patients with intractable temporal lobe epilepsy (TLE) were surgically treated at our institution between 2005 and 2015. A retrospective review of this group identified 44 patients who presented with long-term intractable epilepsy due to a temporal lobe tumour who underwent temporal lobe resections with a postsurgical follow-up of at least two years. Two patients had been previously operated on at the referring institution with unsatisfactory results. The cohort comprised 24 men and 20 women. The age at seizure onset ranged from 2 to 40 years (mean 15.7 years). The mean age at the time of surgery was 30.9 years (range $18-56$ years). The mean duration of epilepsy was 16 years (range 2-34 years).

\section{Preoperative evaluation}

All patients underwent a standard presurgical evaluation that included: history and physical examination with a description of the seizure semiology, serial scalp EEG recording, brain MR imaging at $1.5 \mathrm{~T}$, and a detailed neuropsychological evaluation. In addition, most patients also underwent Wada testing to evaluate for memory and language asymmetry and video-EEG to confirm the localisation and semiology of the seizure. In some patients, a PET study was performed. Invasive subdural monitoring was never used. Cognitive function was tested to measure intellectual quotient and to assess memory, emotional processing, language, and executive function (Wechsler Adult Intelligence Scale-Revised, California Verbal Learning Test, Color Trails Test, Rey-Osterrieth Complex Figure, Tower of London, Boston Naming Test, Verbal Fluency Test). Healthrelated quality of life (HRQoL) was evaluated by completing the QOLIE-31 instrument.
The most common first seizure type was focal impaired awareness (61.3\%). Focal to bilateral tonic-clonic seizures ware noted in 14 cases (41.2\%). Seizures exceeded one per week in 12 patients (27.3\%). Preoperative MR imaging showed typical tumour appearance in all but two cases, in whom the presence of FCD was suspected. Evidence for concomitant mesial temporal sclerosis was not observed, and cortical dysplasia adjacent to the glioneuronal tumours was suspected in five cases. 24 tumours were mesial and 20 were lateral. In 21 patients $(47.7 \%)$, the lesion was on the right side, and in the other 23 patients (52.3\%) it was on the left side. Scalp EEG recordings demonstrated interictal spikes in the ipsilateral temporal lobe in 42 (95.5\%) (eight patients with ipsilateral propagation, 10 patients with bilateral propagation, no propagation in 24 patients), and bilateral interictal spikes in the other two (4.5\%). A minimum of two pre-operative seizures were recorded during video EEG recordings in 38 (86.4\%) patients (in the other six patients with typical seizure history and characteristic temporal lobe tumour appearance on MRI, preoperative seizure recordings were considered unnecessary). In this group, ictal onset recordings demonstrated a theta rhythm in $24(63.2 \%)$ patients, a delta rhythm in $10(26.3 \%)$, and sharp spikes in four (10.5\%). 32 patients underwent a Wada test. In 12 cases of mesial tumour location within the hippocampal complex, a Wada test was not performed. 15 (46.9\%) patients had memory asymmetry on Wada testing compatible with mesial temporal lobe dysfunction. FDG-PET scan was performed in $12(27.3 \%)$ patients and was positive in 11 .

All the clinical data for each patient was then reviewed by a multidisciplinary team (neurosurgeon, epilepsy neurologist, neuroradiologist, and neuropsychologist), and, if appropriate, patients were recommended for surgical therapy.

\section{Surgical procedure}

Total tumour resection along with epileptogenic zone removal were the primary goals of the operation so as to have a better seizure outcome and decrease the chances of recurrence and tumour upgrade. Our surgical strategy comprised tumour resection, and combined mesial temporal and neocortical resection, with varying extents of resection between the two, depending on neuropsychological data, Wada testing, preoperative neurophysiology and intraoperative ECoG testing, and the location of the tumour. Extended lesionectomy with sparing of mesial structures was rarely performed if preoperative Wada testing indicated a possibility of severe memory problems due to contralateral hippocampus memory insufficiency, or if the duration of epilepsy did not exceed five years, or if the tumour was located at the lateral temporal lobe.

After initial electrocorticography (ECoG), tumours located in the lateral temporal lobe and the temporal neocortex were removed first, followed by resection of the deep structures. When located in the mesiotemporal region, tumours were accessed through a transcortical approach with temporal pole resection and further resection of the tumour along the 
amygdala and approximately $3 \mathrm{~cm}$ of the hippocampus. In most cases, a trans-superior temporal gyrus approach was performed, limiting temporal pole resection, with most of the superior temporal gyrus spared. In some cases of left-sided tumour, the superior temporal gyrus was preserved. In general, our temporal lobe resection included the anterior $3.5 \mathrm{~cm}$ of the temporal neocortex in the dominant hemisphere. The anterior lateral temporal neocortex was not removed in a small group of patients in whom mesiotemporal tumour involvement and electro-clinical data indicated a strictly mesial origin of the epileptogenic zone.

Incidental epileptic activity noted on ECoG after temporal lobe resection, evaluated as a result of surgical irritation, was usually not addressed with enhanced surgical resection. However, persistent spiking that produced a broadly spreading field was addressed with further resection in some cases. Neuronavigation technique was not routinely used in temporal lobe epilepsy surgery.

\section{Pathological examination, follow up, and statistical analysis}

All cases were histologically classified according to the CNS [13] and criteria for FCD diagnoses [14, 15]. Surgical and seizure outcomes were assessed in the outpatient epilepsy clinic at six, 12 and 24 months. Postoperative seizure outcome was graded according to the modified Engel classification, as set out in Table 1 [16]. An Engel Class I seizure control outcome

Table 1. Engel Classification

Class I: Free of disabling seizures
IA: Completely seizure-free since surgery
IB: Non-disabling simple partial seizures only since surgery
IC: Some disabling seizures after surgery, but free of disabling seizures
for at least two years
ID: Generalised convulsions with antiepileptic drug withdrawal only
Class II: Rare disabling seizures (almost seizure-free)
A: Initially free of disabling seizures, but rare seizures now
B: Rare disabling seizures since surgery
C: More than rare disabling seizures since surgery, but rare seizures for
the last two years
D: Nocturnal seizures only
Class III: Worthwhile improvement
A: Worthwhile seizure reduction
B: Prolonged seizure-free intervals amounting to more than half of the
follow-up period, but not < 2 years
Class IV: No worthwhile improvement
A: Significant seizure reduction
B: No appreciable change
C: Seizures worse

was considered to be successful treatment, of which the most satisfactory of all was an Engel Class IA outcome. Engel Class II was considered to be an unsatisfactory outcome, while surgical failures were defined as Engel Classes III or IV. Neurological and non-neurological complications were recorded. Antiepileptic medications were managed at the discretion of the treating epileptologist. In order to rule out the presence of any surgical complications in the early postoperative period (up to seven days after surgery), CT scanning with contrast enhancement (with $1.25 \mathrm{~mm}$ of separation acquisition) was routinely carried out. The completeness of tumour resection and the extent of temporal lobe resection were assessed independently by the treating neurosurgeon and neuroradiologist in MR imaging performed in all patients 3-6 months after surgery. Volumetric MRI for quantitative assessment of the completeness of the tumour resection was performed in only a few cases.

The postoperative follow-up period ranged from 2-11 years (mean 5.3 years). HRQoL (QOLIE-31 test) was measured at the two-year follow-up.

The statistical tests used were the Pearson's chi square, paired t-test, Mann-Whitney U test (non-parametric data), and Student's $t$ test (parametric data) for univariate analysis. Univariate and multivariate logistic regression analyses were performed to determine the impact of the independent clinico-pathological variables on seizure freedom status. All statistical analyses were performed using SPSS v.21. A p value $<0.05$ was considered statistically significant.

\section{Results}

\section{Surgical treatment}

Complete tumour removal was performed in all patients. Seven patients underwent limited resection including tumour and peritumoural rim, combined with various degrees of lateral neocortical corticectomy, leaving the amygdala and hippocampus. The remaining 37 patients had complete tumour resection and temporal pole and lateral neocortex removal along with the hippocampus. Persistent temporal spiking noted on ECoG after resection was addressed with further resection in four patients, and in only one case was it still present.

\section{Pathological findings}

The histological examination revealed that glioneuronal tumours (32 patients, 72.7\%) were more common than low-grade gliomas (12 patients, 27.3\%), as set out in Table 2. DNT (dysembryoplastic neuroepithelial tumour) was the most frequent diagnosis, found in 17 patients (38.6\%). Interestingly, two cases (4.5\%) of anaplastic tumours had a seven-year history of intractable seizures. Concomitant ipsilateral mesial temporal sclerosis was noted in one patient (2.3\%), indicative of dual pathology, even though there was no evidence for MTS on preoperative MRI. Furthermore, histological examination in $19(43.2 \%)$ cases showed FCD, namely four cases of FCD 
Table 2. Pathological findings in 44 tumour epilepsy-related patients

\begin{tabular}{lc}
\multicolumn{1}{c}{ Tumour type } & No. of patients (\%) \\
\hline DNT (WHO GI) & $17(38.6)$ \\
Ganglioglioma (WHO GI) & $14(31.8)$ \\
Ganglioglioma anaplasticum (WHO GIII) & $1(2.3)$ \\
Astrocytoma pilocyticum (WHO GI) & $5(11.4)$ \\
Oligodendroglioma (WHO GII) & $2(4.5)$ \\
Pleomorphic xanthoastrocytoma (WHO GII) & $2(4.5)$ \\
Astrocytoma fibryllare (WHO GII) & $1(2.3)$ \\
Atrocytoma anaplasticum (WHO GIII) & $1(2.3)$ \\
Angiocentric glioma (WHO GI) & $1(2.3)$
\end{tabular}

Type IIA and 15 cases of FCD Type IIIB. All but one FCDs were associated with glioneuronal tumours.

\section{Surgical outcome and complications}

No patient died during surgery or the postoperative course. Seven (13.6\%) patients had postoperative neurological complications. Two (4.5\%) of them had permanent hemiparesis due to ischaemic stroke (in one case hemiparesis was accompanied by dysphasia and $3^{\text {rd }}$ cranial nerve deficit). In the other five patients, transient postoperative complications were noted and included: temporary dysphasia in two patients, transitory hemiparesis in one, postoperative haematoma in one, and intracranial hypotension syndrome (hypoperfusion syndrome) in one. In eight (18.2\%) patients, significant new field cuts (hemianopsia) were observed.

MRI showed evidence of tumour recurrence in the patient with anaplastic ganglioglioma (GG). This patient was treated oncologically and died six years after surgery. The other patient with anaplastic astrocytoma, after postoperative radiotherapy underwent a second craniotomy because of radiographic suspicion of tumour recurrence. However, no tumour was identified on pathology, which showed only the presence of radiation necrosis.

\section{Seizure outcome}

At the final follow-up, favourable seizure outcome (Engel I) was found in 37 patients (84\%), including 31 (70.5\%) in Engel Class IA (excellent result). Two (4.5\%) patients presented with an Engel Class II outcome (unfavourable outcome). Five patients (11.5\%) were in Engel Classes III or IV (surgical failure).

Statistical comparisons of the various clinical, radiological, perioperative, and pathological factors in patients with an Engel Class I outcome and patients with Engel Classes II-IV outcomes are set out in Table 3 , and prognostic indicators are identified.

Four parameters correlated with the rate of seizure freedom after surgery: extent of temporal lobe resection $(\mathrm{p}=0.001)$, postoperative ECoG findings ( $\mathrm{p}=0.022)$, and pathological diagnosis and tumour-associated FCD occurrence $(\mathrm{p}=0.004$ and $p=0.012$, respectively). Of the seven patients who underwent lesionectomy, three were in Engel Class I (42.9\%), one was in
Engel Class II, and three were in Engel Classes III-IV. Among individuals who had complete resection of the hippocampus along with tumour and extended temporal pole removal, the postoperative seizure freedom rate was $91.9 \%$ (34 were Engel Class I, one was Engel Class II, and two were Engel Classes III-IV).

Pathological diagnosis was also a significant prognostic indicator of seizure freedom. Patients with a diagnosis of a glioneuronal tumour benefited from a $93.8 \%$ rate of seizure freedom after resection, compared to only $58.3 \%$ of those who had a low-grade glioma. Moreover, tumour-associated FCDs significantly correlated with a satisfactory seizure outcome.

The use of intraoperative ECoG was found to influence the outcome. Of 30 patients who presented with no epileptogenic discharges after resection, Engel Class I outcome was obtained in 28 (93.3\%). In contrast, Engel Class I was seen in 6/8 (75\%) patients with sharp spikes occurring postoperatively. However, intraoperative ECoG was not used in six patients of whom only three $(50 \%)$ were free of seizures after surgery.

Table 4 shows how logistic regression analyses were used for further scrutiny of the four variables found on chi-square testing to prognosticate significant factors for seizure outcome. We found that the extent of resection produced the biggest difference in postoperative seizure status: complete resection of the hippocampus along with tumour and extended temporal pole removal was strongly associated with seizure freedom $(\mathrm{p}$ $=0.015$ ). This implies that resection sparing the hippocampal-parahippocampal complex could greatly increase the risk of persistent seizures. Furthermore, we found that pathological diagnosis independently predicted postoperative seizure status: glioneuronal tumours were associated with a better seizure outcome $(\mathrm{p}=0.024)$.

\section{Quality of life outcome}

There was a statistically significant improvement for the overall QOLIE-31 score $(46.73+/-21.16$ before surgery and $69.31+/-29.17$ after surgery, $\mathrm{p}<0.005)$. However, there was no statistically significant effect of epilepsy surgery on HRQoL changes between the various subgroups based on the Engel class surgical outcome.

\section{Discussion}

Chronic medically intractable epilepsy secondary to low-grade temporal tumours is well known [17]. Advances in imaging and neuropathological techniques have allowed such tumours to be detected with increasing frequency before surgery [18].

In determining the most appropriate treatment for patients with tumoural epilepsy, it is important to understand the natural history of this disease and its treatment options and surgical outcomes. Usually epilepsy associated with low-grade tumours is insufficiently controlled by antiepileptic drugs, but is extremely responsive to surgical treatment.

It should be emphasised that in most of these patients, epilepsy control is the main goal of surgery because most are 

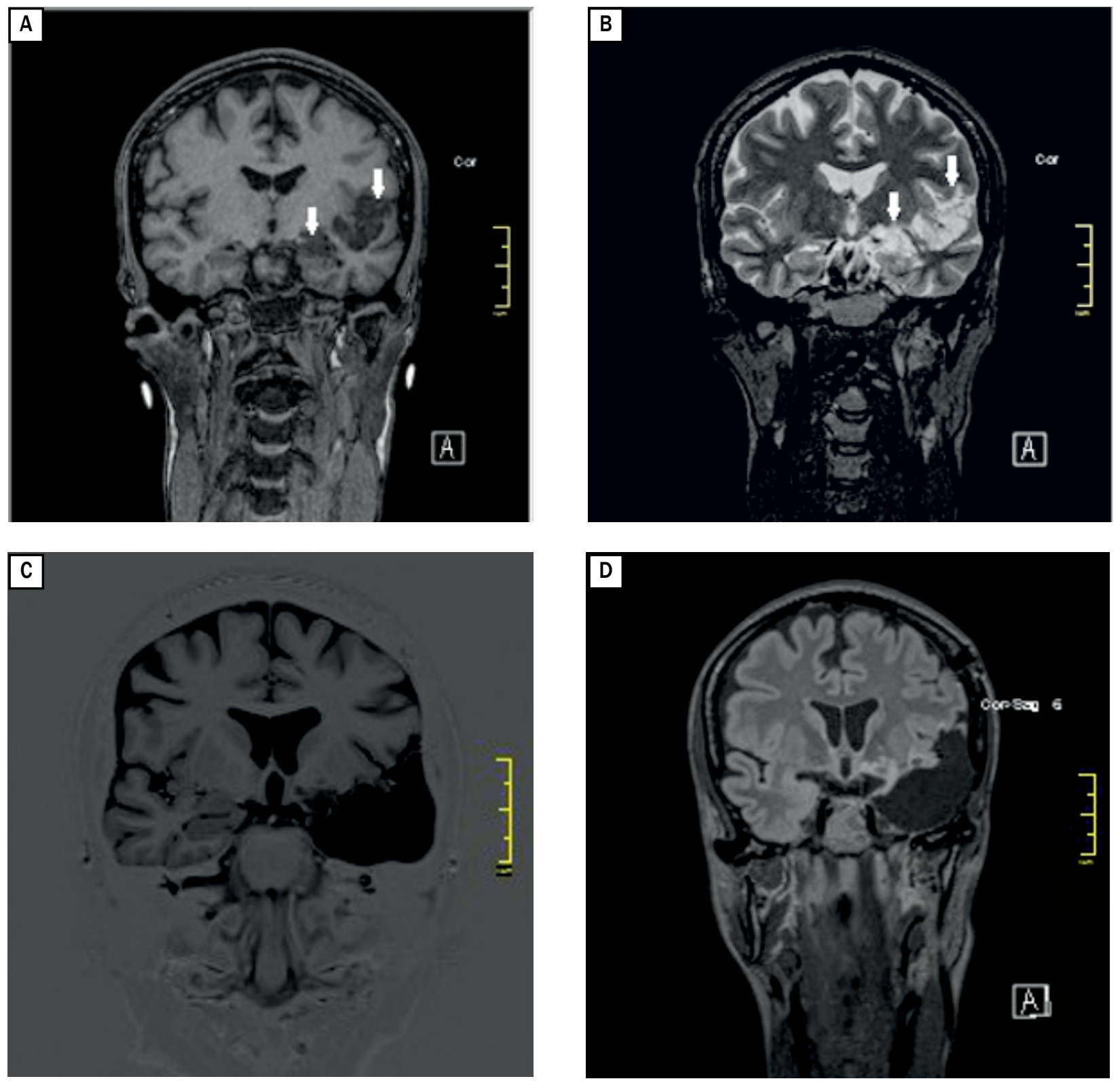

Figure 1. Preoperative coronal T1 (A) and coronal T2 (B) MR image demonstrating extensive DNT associated with FCD type IIA of the left temporal lobe (white arrows). Postoperative coronal Inversion Recovery (IR) (C) and coronal FLAIR (D) MR image obtained after temporal lobe resection including tumour, lateral neocortex and hippocampal complex removal

benign tumours of glioneuronal or glial origin [19]. However, in those with a diffuse low-grade glioma (WHO GII), the oncological aspects of treatment should prevail according to the progressive course of these tumours.

In this report, we have presented our experience in 44 cases involving chronic intractable epilepsy associated with temporal lobe tumours, and we have discussed our management and outcomes.

\section{Pathological diagnosis}

It has been previously reported that glioneuronal tumours and low-grade gliomas account for the great majority of tumours in patients with chronic epilepsy [20]. These are generally benign and indolent tumours in which epilepsy is usually the main problem, although the rare occurrence of malignant evolution, as in two of our cases, has been reported.

Among the published series, however, there is no consensus regarding which histological entity is the most common. In our series, DNT was the most common diagnosis, consistent with other reports [21]. Other groups have reported ganglioglioma [22] and astrocytoma [3] to be the most common. Gangliogliomas and DNTs may have intrinsic epileptogenic properties due to their neuronal and glial components, and 
Table 3. Comparison of demographic, clinical and pathological characteristics of patients with tumour-related temporal lobe epilepsy between those who are seizure-free and those with persistent seizures

\begin{tabular}{|c|c|c|c|}
\hline Variables & $\begin{array}{c}\begin{array}{c}\text { Seizure free } \\
(n=37)\end{array} \\
\text { No. of patients (\%) }\end{array}$ & $\begin{array}{l}\text { Persistent seizures }(n=7) \\
\text { No. of patients }(\%)\end{array}$ & $P$ value \\
\hline Sex, male/female* & $21(56.8) / 16(43.2)$ & $3(42.9) / 4(57.1)$ & 0.498 \\
\hline Initial precipitating injury, yes/no* & $9(24.3) / 28(75.7)$ & $2(28.6) / 5(71.4)$ & 0.812 \\
\hline $\begin{array}{l}\text { Type of first seizure, tonic-clonic / focal impaired awareness / } \\
\text { focal aware* }\end{array}$ & $11(29.7) / 22(59.5) / 4(10.8)$ & $2(28.6) / 5(71.4) / 0(0)$ & 0.639 \\
\hline Mean age at surgery (SD), years** & $30.92(+/-8.496)$ & $33.14(+/-11.992)$ & 0.555 \\
\hline Mean age at seizure onset (SD), years** & $15.97(+/-8.241)$ & $13.71(+/-7.064)$ & 0.502 \\
\hline Mean duration of epilepsy (SD), years** & $14.73(+/-8.061)$ & $18.29(+/-10.029)$ & 0.309 \\
\hline Number of seizures per week (preop), $<1 />=1^{*}$ & $26(70.3) / 11(29.7)$ & $6(85.7) / 1(14.3)$ & 0.400 \\
\hline Focal to bilateral tonic-clonic seizures, yes/no* & $11(29.7) / 26(70.3)$ & $3(42.9) / 4(57.1)$ & 0.494 \\
\hline Propagation of focal changes on scalp EEG, yes/no* & $15(40.5) / 22(59.5)$ & $3(42.9) / 4(57.1)$ & 0.909 \\
\hline $\begin{array}{l}\text { Ictal onset recordings on video-EEG, theta/delta/sharp spikes/ } \\
\text { data lacking* }\end{array}$ & $\begin{array}{c}7(18.9) / 22(59.5) / 4(10.8) / \\
4(10.8)\end{array}$ & $3(42.9) / 2(28.6) / 0(0) / 2(28.6)$ & 0.201 \\
\hline Tumour location, mesial/lateral* & $22(59.5) / 15(40.5)$ & $2(28.6) / 5(71.4)$ & 0.132 \\
\hline Side of surgery, right/left* & $17(45.7) / 20(54.1)$ & $4(57.1) / 3(42.9)$ & 0.587 \\
\hline Extent of temporal lobe resection, lesionectomy/complete $T L R^{*}$ & $3(8.1) / 34(91.9)$ & $4(57.1) / 3(42.9)$ & 0.001 \\
\hline $\begin{array}{l}\text { Postoperative ECoG findings, sharp spikes/slow waves/data } \\
\text { lacking* }\end{array}$ & $6(16.2) / 28(75.7) / 3(8.1)$ & $2(28.6) / 2(28.6) / 3(42.9)$ & 0.022 \\
\hline Seizure in early post-op period, yes $/ \mathrm{no}^{*}$ & $7(18.9) / 30(81.1)$ & $1(14) / 6(85.7)$ & 0.771 \\
\hline Post-op complications, yes $/ \mathrm{no}^{*}$ & $10(27) / 27(73)$ & $0(0) / 7(100)$ & 0.118 \\
\hline Tumour pathology, glioneuronal/glial* & $30(81.1) / 7(18.9)$ & $2(28.6) / 5(71.4)$ & 0.004 \\
\hline Tumour-associated FCD occurrence, yes/no* & $19(51.4) / 18(48.6)$ & $0(0) / 7(100)$ & 0.012 \\
\hline
\end{tabular}

*Pearson's chi square test, ${ }^{* *} \mathrm{t}$ Student test, TLR - temporal lobe resection, FCD - focal cortical dysplasia

Table 4. Predictors of seizure class I outcome (logistic regression model)

\begin{tabular}{llcc} 
Variables & Wald & Exp (B) (95\% Cl) & P value \\
\hline Extent of temporal lobe resection, lesionectomy/complete TLR & 5.953 & $0.47(0.004-0.547)$ & 0.015 \\
Postoperative ECoG findings, sharp spikes/slow waves/data lacking & 1.134 & $2.733(0.429-17.397)$ & 0.287 \\
Tumour pathology, glioneuronal/glial & 5.061 & $14.867(1.416-156.136)$ & 0.024 \\
Tumour-associated FCD occurrence, yes/no & 2.355 & $0.136(0.11-1.738)$ & 0.125
\end{tabular}

the precise epileptological mechanisms associated with these lesions are still under investigation [7, 23-25].

Of importance is that our report confirms the high incidence of tumour-associated cortical dysplasia (43.2\%) and the presence of cases of mesial temporal sclerosis associated with neoplasm.

The above observations have led us to adopt the strategy of extended temporal lobe resection in cases of tumoural drug-resistant epilepsy. Glioneuronal tumours are associated with FCD in $30-80 \%$ of cases [24-26], and have been considered by some authors as the extreme neoplastic end of the histopathological spectrum of cortical dysplasia $[8,15,27]$. The new ILAE classification system for FCDs introduced FCD type IIIB to encapsulate cases of FCD type I adjacent to a low-grade tumour associated with epilepsy [14]. However, the specific role of each of them in epileptogenesis is still not fully defined $[8,15,27]$.

While rare, there have been reports of anaplastic transformation of gangliogliomas [28]. In our series, there were two cases of anaplastic tumours. Both of them had a seven-year history of intractable seizures, suggesting the possibility of anaplastic transformation.

\section{Surgical treatment and seizure outcome}

Temporal lobe resection is the long-established safe and effective treatment for temporal lobe epilepsy [29]. In those patients who harbour temporal lobe tumours however, there has been considerable controversy regarding the most appropriate management, with some advocating lesionectomy only, and others arguing for more extensive resection [17, 20,30]. 

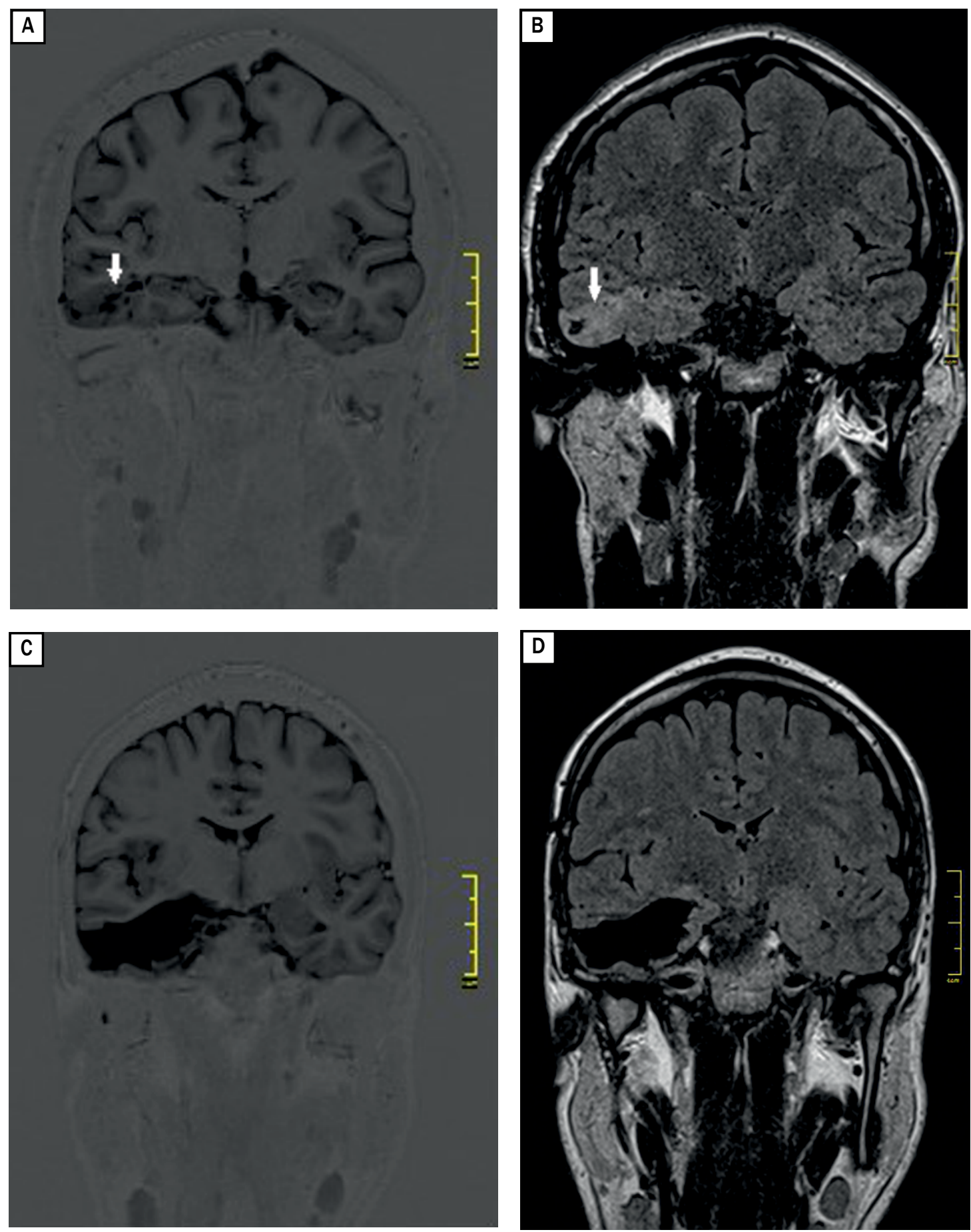

Figure 2. Preoperative coronal Inversion Recovery (IR) (A) and coronal FLAIR (B) MR image demonstrating ganglioglioma associated with FCD type IIIB of the right temporal lobe (white arrows). Postoperative coronal Inversion Recovery (IR) (C) and coronal FLAIR (D) MR image obtained after tailored temporal lobe resection including tumour, temporal pole and hippocampal complex removal

Because it is unclear how much the peritumoural tissue contributes to the initiation and propagation of seizures, the extent of resection that must be performed is not obvious [10]. The only consensus appears to be that gross-total resection, as long as it can be safely performed, should be the minimum goal of surgery $[17,31,32]$. It seems that temporal low-grade tumours, especially those of glioneuronal origin located in the mesiotemporal region, are a distinct clinico-pathological group with a more widespread epileptic network and complex epileptogenic mechanisms. Here, tailored resection appears to offer the best results for seizure outcome [26, 33-35]. Lesionectomy is considered sufficient for an optimal seizure outcome 
in temporolateral and extratemporal low-grade tumours [33]. In our series, our surgical strategy was independent of the histological type expected. The extent of resection was based on identification of the epileptogenic area and determined by noninvasive presurgical clinical and neurophysiological investigations.

In addition to gross-total removal of the tumour, $84 \%$ of patients underwent resection of the amygdala and hippocampus. We found that the extent of resection led to the biggest difference in postoperative seizure status.

Resection of the hippocampal-parahippocampal complex in addition to the tumour, especially when it is not involved in the lesion and appears normal on MRI, is controversial. However, it is well known that the mesial temporal structures have a great epileptogenicity, and their resection is considered crucial for surgical success in refractory mesial temporal lobe epilepsy, because both the lesion and the hippocampus are epileptogenic.

We found that our approach of extended temporal lobe resection provided for optimal resection of epileptogenic tissue by removing both mesial temporal structures and invisible foci of highly epileptogenic cortical dysplasia adjacent to the neoplasm. Although a clear relationship between epilepsy and tumour-associated FCD has not been fully established, it has been reported that such an association may determine a less effective control of seizures after simple lesionectomy $[7,8$, $24,36]$. Glioneuronal tumours are frequently embedded in dysplastic cortex, which is more likely to be removed when performing a tailored resection [26]. There is also no agreement on the best time for surgery, as these tumours may not show significant growth for many years.

The decision to proceed to surgery in our patients was based on the fact that epilepsy associated with low-grade tumours is a typical example of surgically-remediable epilepsy with an excellent seizure outcome, and effective surgical treatment might avoid possible drug-induced adverse cognitive effects $[7,9,11,21,26]$. Moreover, epilepsy surgery may also prevent the possibility of malignant transformation $[7,9,11,12,24]$.

The patients in our series experienced significantly improved outcomes with respect to seizure frequency. These results are in line with other similar series, in which more than $80 \%$ of patients were free of disabling seizures after surgery $[3,4$, $17,22]$. We found that low-grade tumour type (glioneuronal versus low-grade glioma) predicted seizure freedom after surgery. This might be explained by the fact that gross-total tumour resection is the strongest predictor for favourable seizure outcome [11,34, 37-39]. However, it may prove difficult in cases of low-grade gliomas. WHO Grade II low-grade gliomas could present in locations that are challenging to access surgically and involve the paralimbic system. Furthermore, even after presumed total tumour resection, there are residual tumour cells acting as an epileptic focus, since it has been demonstrated that gliomas involve the brain beyond the signal abnormality visible on MRI [40].
In our series, there were no statistical differences between clinical factors consistent with epilepsy severity and seizure outcome. It has been reported that strong predictors of seizure freedom after surgery are short duration of epilepsy and lack of focal to bilateral seizures before surgery [34, 41]. Shorter epilepsy duration has been shown to predict improved seizure outcome in systematic reviews examining gangliogliomas and low-grade gliomas [37, 42]. However, in patients with temporal lobe epilepsy, epilepsy duration has not been found to be predictive of its severity [43]. The use of intraoperative ECoG has been reported not to be associated with improved seizure outcomes, which is consistent with our findings. But caution in interpreting this finding is advised, because ECoG may only be used in more severe cases [34].

The rates of permanent new neurological deficits in other series are comparable to ours [44]. Temporal lobe lesions carry a particular risk for the visual pathways, although postoperative quadrantanopia is considered not to be a significant complication, instead being seen as a non-disturbing acceptable side effect [45].

Outcome measures have been focused mainly on seizure relief, but it is also necessary to look at outcomes related to psychosocial functioning and health-related quality of life (HRQoL) [46]. Postoperative improvement in psychosocial functioning has been shown, although the small number of subjects in Engel Class I, and Engel Classes II-IV surgical outcomes, limited the power to detect a difference between the subgroups.

\section{Conclusions}

The most appropriate management of tumour-related chronic temporal lobe epilepsy in adults appears to be tailored temporal lobe resection including tumour and hippocampal complex removal. Surgical treatment of tumoural temporal lobe epilepsy demonstrates excellent results with seizure improvement, especially in patients with glioneuronal tumours.

Funding: This publication was prepared without any external source of funding.

Conflicts of interest: None.

Ethical approval was not necessary for preparation of this article.

\section{References}

1. Klein M, Engelberts $\mathrm{NHJ}$, van der Ploeg HM, et al. Epilepsy in lowgrade gliomas: the impact on cognitive function and quality of life. Ann Neurol. 2003; 54(4): 514-520, doi: 10.1002/ana.10712, indexed in Pubmed: 14520665.

2. Taphoorn MJB, Klein M. Cognitive deficits in adult patients with brain tumours. Lancet Neurol. 2004; 3(3): 159-168, doi: 10.1016/S14744422(04)00680-5, indexed in Pubmed: 14980531.

3. Zaatreh MM, Firlik KS, Spencer DD, et al. Temporal lobe tumoral epilepsy: characteristics and predictors of surgical outcome. Neurology. 2003; 61(5): 636-641, doi: 10.1212/01.wnl.0000079374.78589.1b, indexed in Pubmed: 12963754. 
4. Iannelli A, Guzzetta F, Battaglia D, et al. Surgical treatment of temporal tumors associated with epilepsy in children. Pediatr Neurosurg. 2000; 32(5): 248-254, doi: 10.1159/000028946, indexed in Pubmed: 10965271.

5. Bourgeois M, Di Rocco F, Sainte-Rose C. Lesionectomy in the pediatric age. Childs Nerv Syst. 2006; 22(8): 931-935, doi: 10.1007/s00381 006-0146-z, indexed in Pubmed: 16821076.

6. Giulioni M, Galassi E, Zucchelli M, et al. Seizure outcome of lesionectomy in glioneuronal tumors associated with epilepsy in children. J Neurosurg. 2005; 102(3 Suppl): 288-293, doi: 10.3171/ ped.2005.102.3.0288, indexed in Pubmed: 15881752.

7. Aronica E, Leenstra S, van Veelen CW, et al. Glioneuronal tumors and medically intractable epilepsy: a clinical study with long-term follow-up of seizure outcome after surgery. Epilepsy Res. 2001; 43(3): 179191, doi: 10.1016/s0920-1211(00)00208-4, indexed in Pubmed: 11248530.

8. Chan CH, Bittar RG, Davis GA, et al. Long-term seizure outcome following surgery for dysembryoplastic neuroepithelial tumor. J Neurosurg. 2006; 104(1): 62-69, doi: 10.3171/jns.2006.104.1.62, indexed in Pubmed: 16509148.

9. Cossu M, Lo Russo G, Francione S, et al. Epilepsy surgery in children: results and predictors of outcome on seizures. Epilepsia. 2008; 49(1): 65-72, doi: 10.1111/j.1528-1167.2007.01207.x, indexed in Pubmed: 17645538.

10. Jooma R, Yeh HS, Privitera MD, et al. Lesionectomy versus electrophysiologically guided resection for temporal lobe tumors manifesting with complex partial seizures. J Neurosurg. 1995; 83(2): 231-236, doi: 10.3171/jns.1995.83.2.0231, indexed in Pubmed: 7616267.

11. Luyken C, Blümcke I, Fimmers R, et al. The spectrum of long-term epilepsy-associated tumors: long-term seizure and tumor outcome and neurosurgical aspects. Epilepsia. 2003; 44(6): 822-830, doi: 10.1046/j.1528-1157.2003.56102.x, indexed in Pubmed: 12790896.

12. Luyken C, Blümcke I, Fimmers R, et al. Supratentorial gangliogliomas: histopathologic grading and tumor recurrence in 184 patients with a median follow-up of 8 years. Cancer. 2004; 101(1): 146-155, doi: 10.1002/cncr.20332, indexed in Pubmed: 15222000.

13. Louis DN, Ohgaki H, Wiestler OD, et al. The 2007 WHO classification of tumours of the central nervous system. Acta Neuropathol. 2007; 114(2): 97-109, doi: 10.1007/s00401-007-0243-4, indexed in Pubmed: 17618441.

14. Blümcke I, Thom M, Aronica E, et al. The clinicopathologic spectrum of focal cortical dysplasias: a consensus classification proposed by an ad hoc Task Force of the ILAE Diagnostic Methods Commission. Epilepsia. 2011; 52(1): 158-174, doi: 10.1111/j.1528-1167.2010.02777.x, indexed in Pubmed: 21219302.

15. Palmini A, Najm I, Avanzini G, et al. Terminology and classification of the cortical dysplasias. Neurology. 2004; 62(6 Suppl 3): S2-S8, doi: 10.1212/01.wnl.0000114507.30388.7e, indexed in Pubmed: 15037671.

16. Fischer-Williams M. Surgical treatment of the epilepsies, 2nd edition. Electroencephalography and Clinical Neurophysiology. 1994; 90(3): 249-250, doi: 10.1016/0013-4694(94)90099-x.

17. Britton JW, Cascino GD, Sharbrough FW, et al. Low-grade glial neoplasms and intractable partial epilepsy: efficacy of surgical treatment. Epilepsia. 1994; 35(6): 1130-1135, doi: 10.1111/j.15281157.1994.tb01778.x, indexed in Pubmed: 7988500.

18. Bernasconi A, Bernasconi N, Bernhardt BC, et al. Advances in MRI for 'cryptogenic' epilepsies. Nat Rev Neurol. 2011; 7(2): 99-108, doi: 10.1038/nrneurol.2010.199, indexed in Pubmed: 21243016.
19. Vogt VL, Witt JA, Delev D, et al. Cognitive features and surgical outcome of patients with long-term epilepsy-associated tumors (LEATs) within the temporal lobe. Epilepsy Behav. 2018; 88: 25-32, doi: 10.1016/j.yebeh.2018.08.028, indexed in Pubmed: 30212725.

20. Berger MS, Ghatan S, Haglund MM, et al. Low-grade gliomas associated with intractable epilepsy: seizure outcome utilizing electrocorticography during tumor resection. J Neurosurg. 1993; 79(1): 62-69, doi: 10.3171/jns.1993.79.1.0062, indexed in Pubmed: 8315470.

21. Cataltepe 0, Turanli G, Yalnizoglu D, et al. Surgical management of temporal lobe tumor-related epilepsy in children. J Neurosurg. 2005; 102(3 Suppl): 280-287, doi: 10.3171/ped.2005.102.3.0280, indexed in Pubmed: 15881751.

22. Ruban D, Byrne RW, Kanner A, et al. Chronic epilepsy associated with temporal tumors: long-term surgical outcome. Neurosurg Focus. 2009; 27(2): E6, doi: 10.3171/2009.5.FOCUS0998, indexed in Pubmed: 19645562.

23. Aronica E, Redeker S, Boer K, et al. Inhibitory networks in epilepsy-associated gangliogliomas and in the perilesional epileptic cortex. Epilepsy Res. 2007; 74(1): 33-44, doi: 10.1016/j.eplepsyres.2006.12.002, indexed in Pubmed: 17267178.

24. Blümcke I, Wiestler OD. Gangliogliomas: an intriguing tumor entity associated with focal epilepsies. J Neuropathol Exp Neurol. 2002; 61(7): 575-584, doi: 10.1093/jnen/61.7.575, indexed in Pubmed: 12125736 .

25. Chassoux F, Daumas-Duport C. Dysembryoplastic neuroepithelial tumors: where are we now? Epilepsia. 2013; 54 Suppl 9: 129-134, doi: 10.1111/epi.12457, indexed in Pubmed: 24328886.

26. Giulioni M, Rubboli G, Marucci G, et al. Seizure outcome of epilepsy surgery in focal epilepsies associated with temporomesial glioneuronal tumors: lesionectomy compared with tailored resection. J Neurosurg. 2009; 111(6): 1275-1282, doi: 10.3171/2009.3.JNS081350, indexed in Pubmed: 19408976.

27. Becker AJ, Blümcke I, Urbach $\mathrm{H}$, et al. Molecular neuropathology of epilepsy-associated glioneuronal malformations. J Neuropathol Exp Neurol. 2006; 65(2): 99-108, doi: 10.1097/01. jnen.0000199570.19344.33, indexed in Pubmed: 16462201.

28. Sasaki A, Hirato J, Nakazato Y, et al. Recurrent anaplastic ganglioglioma: pathological characterization of tumor cells. Case report. J Neurosurg. 1996; 84(6): 1055-1059, doi: 10.3171/jns.1996.84.6.1055, indexed in Pubmed: 8847572.

29. Wiebe S, Blume WT, Girvin JP, et al. Effectiveness and Efficiency of Surgery for Temporal Lobe Epilepsy Study Group. A randomized, controlled trial of surgery for temporal-lobe epilepsy. N Engl J Med. 2001; 345(5): 311-318, doi: 10.1056/NEJM200108023450501, indexed in Pubmed: 11484687.

30. Chong S, Phi JiH, Lee JiY, et al. Surgical Treatment of Lesional Mesial Temporal Lobe Epilepsy. J Epilepsy Res. 2018; 8(1): 6-11, doi: 10.14581/jer.18002, indexed in Pubmed: 30090756.

31. Yaşargil MG, Krayenbühl N, Roth $P$, et al. The selective amygdalohippocampectomy for intractable temporal limbic seizures. J Neurosurg. 2010; 112(1): 168-185, doi: 10.3171/2008.12.JNS081112, indexed in Pubmed: 19575575.

32. Ko A, Kim SeH, Kim SeH, et al. Epilepsy Surgery for Children With Low-Grade Epilepsy-Associated Tumors: Factors Associated With Seizure Recurrence and Cognitive Function. Pediatr Neurol. 2019; 91: 50-56, doi: 10.1016/j.pediatrneurol.2018.10.008, indexed in Pubmed: 30477743.

33. Babini M, Giulioni M, Galassi E, et al. Seizure outcome of surgical treatment of focal epilepsy associated with low-grade tumors in children. 
J Neurosurg Pediatr. 2013; 11(2): 214-223, doi: 10.3171/2012.11. PEDS12137, indexed in Pubmed: 23215740.

34. Englot DJ, Berger MS, Barbaro NM, et al. Factors associated with seizure freedom in the surgical resection of glioneuronal tumors. Epilepsia. 2012; 53(1): 51-57, doi: 10.1111/j.1528-1167.2011.03269.x, indexed in Pubmed: 21933181.

35. Abel TJ, Woodroffe RW, Nourski KV, et al. Role of the temporal pole in temporal lobe epilepsy seizure networks: an intracranial electrode investigation. J Neurosurg. 2018; 129(1): 165-173, doi: 10.3171/2017.3.JNS162821, indexed in Pubmed: 29027858.

36. Josephson CB, Dykeman J, Fiest KM, et al. Systematic review and meta-analysis of standard vs selective temporal lobe epilepsy surgery. Neurology. 2013; 80(18): 1669-1676, doi: 10.1212/ WNL.0b013e3182904f82, indexed in Pubmed: 23553475.

37. Englot DJ, Berger MS, Barbaro NM, et al. Predictors of seizure freedom after resection of supratentorial low-grade gliomas. A review. J Neurosurg. 2011; 115(2): 240-244, doi: 10.3171/2011.3.JNS1153, indexed in Pubmed: 21529134.

38. Grote A, Witt JA, Surges R, et al. A second chance-reoperation in patients with failed surgery for intractable epilepsy: long-term outcome, neuropsychology and complications. J Neurol Neurosurg Psychiatry. 2016; 87(4): 379-385, doi: 10.1136/jnnp-2015-310322, indexed in Pubmed: 25855399.

39. Giulioni M, Marucci G, Pelliccia V, et al. Commission for Epilepsy Surgery of the Italian League Against Epilepsy. Epilepsy surgery of "low grade epilepsy associated neuroepithelial tumors": A retrospective nationwide Italian study. Epilepsia. 2017; 58(11): 1832-1841, doi: 10.1111/epi.13866, indexed in Pubmed: 28804898.

40. Pallud J, Varlet P, Devaux B, et al. Diffuse low-grade oligodendrogliomas extend beyond MRI-defined abnormalities. Neurology. 2010;
74(21): 1724-1731, doi: 10.1212/WNL.0b013e3181e04264, indexed in Pubmed: 20498440.

41. Daszkiewicz P, Kowalczyk P, Roszkowski M. Surgical treatment of neuronal-glial tumors of mesial-basal part of temporal lobe: Long term outcome and control of epilepsy in pediatric patients. Neurol Neurochir Pol. 2018; 52(1): 2-8, doi: 10.1016/j.pjnns.2017.04.001, indexed in Pubmed: 29029800.

42. Yang I, Chang EF, Han SJ, et al. Early surgical intervention in adult patients with ganglioglioma is associated with improved clinical seizure outcomes. J Clin Neurosci. 2011; 18(1): 29-33, doi: 10.1016/j. jocn.2010.05.002, indexed in Pubmed: 20961765.

43. Lowe NM, Eldridge P, Varma T, et al. The duration of temporal lobe epilepsy and seizure outcome after epilepsy surgery. Seizure. 2010; 19(5): 261-263, doi: 10.1016/j.seizure.2010.02.011, indexed in Pubmed: 20430656.

44. Hader WJ, Tellez-Zenteno J, Metcalfe A, et al. Complications of epilepsy surgery: a systematic review of focal surgical resections and invasive EEG monitoring. Epilepsia. 2013; 54(5): 840-847, doi: 10.1111/ epi.12161, indexed in Pubmed: 23551133.

45. Schramm J, Aliashkevich AF, Schramm J, et al. Surgery for temporal mediobasal tumors: experience based on a series of 235 patients. Neurosurgery. 2007; 60(2): 285-94; discussion 294, doi: 10.1227/01.NEU.0000249281.69384.D7, indexed in Pubmed: 17290179.

46. Helmstaedter C, Elger CE, Vogt VL. Cognitive outcomes more than 5 years after temporal lobe epilepsy surgery: Remarkable functional recovery when seizures are controlled. Seizure. 2018; 62: 116-123, doi: 10.1016/j.seizure.2018.09.023, indexed in Pubmed: 30359865 . 